\title{
High Performance Computer Methods Applied to Predictive Space Weather Simulations
}

\author{
C. Robert Clauer, Tamas I. Gombosi, Darren L. De Zeeuw, Aaron J. Ridley, Kenneth G. Powell, Bram van Leer, \\ Quentin F. Stout, Clinton P. T. Groth, and Thomas E. Holzer
}

\begin{abstract}
Taking advantage of the advent of massively parallel computers, sophisticated solution-adaptive techniques, and recent fundamental advances in basic numerical methods we have developed a high performance, adaptive-scale MHD code capable of resolving many of the critical processes in the Sun-Earth system which range over more than nine orders of magnitude. The development of such models are of increasing importance as the impact of space weather on vulnerable technklogical systems increases, and too, as the severity of space weather increases with the approach of solar maximum. There is an increasing need to develop physics- based, high performance models of the Sun-Earth system from the solar surface to the Earth's upper atmosphere which can operate faster than real time and which can provide reliable predictions of the near Earth space environment based upon solar observations and upstream solar wind measurements. We report on the status of the Michigan adaptive-scale MHD model, which is one effort whose goal is the development of an operational predictive space weather model.
\end{abstract}

Index Terms-Adaptive mesh refinement, magnetohydrodynamic, numerical simulation, space weather.

\section{INTRODUCTION}

$\mathbf{T}$ HE Sun-Earth system is an extremely complex natural system involving many different interacting elements. Today, the science of space plasma physics has matured to the level of being able to describe many of these complex interactions and to model them. A major goal now is to unify our understanding into a more comprehensive mathematical framework that can simulate and predict the properties of this system (space weather).

This goal is made all the more relevant and timely because of the increased need for a predictive space weather model. "Space Weather" has been used to refer to the conditions on the Sun and in the solar wind, magnetosphere, ionosphere, and thermosphere that can influence the performance and reliability

Manuscript received December 1, 1999; revised April 21, 2000. This work was supported in part by NSF Knowledge and Distributed Intelligence Grant ATM-9980078, NASA HPCC CAN NCCS5-146, and a NSF-NASA-AFOSR Interagency Grant NSF-ATM-9318181.

C. R. Clauer, T. I. Gombosi, D. L. De Zeeuw, and A. J. Ridley are with the Space Physics Research Laboratory, University of Michigan, Ann Arbor, MI USA (e-mail: rclauer@umich.edu).

K. G. Powell and B. van Leer are with the Department of Aerospace Engineering, University of Michigan, Ann Arbor, MI USA.

Q. F. Stout is with the Department of Electrical Engineering and Computer Science at the University of Michigan, Ann Arbor, MI USA.

C. P. T. Groth is with the Institute of Aerospace Studies, University of Toronto, North York, Ontario, Canada.

T. E. Holzer is with the High Altitude Observatory, National Center for Atmospheric Research, Boulder, CO USA.

Publisher Item Identifier S 0093-3813(00)10652-6. of space-borne and ground-based technological systems or can endanger human life or health. For example, satellites experience the disruptive effects of energetic particles and differential electrical charging during various conditions; astronauts are vulnerable to energetic radiation that may occur at space station altitudes; navigation signals from the GPS satellites are affected by irregularities in the ionosphere that occur under some conditions; massive disruptions in electric power distribution systems can be triggered by geomagnetic storms; and the Skylab space station crashed into the atmosphere because of the increased atmospheric drag due to the atmospheric heating and consequent expansion which resulted from several unanticipated severe geomagnetic storms; to name a few consequences of space weather.

To build a predictive, physics-based, space weather computer model, however, requires that we address and solve several difficult problems. It is necessary to integrate and synthesize the work of many scientists in many disciplines. Further, translating this understanding into models capable of representing the large range of scales for critical phenomena in this system requires very advanced numerical technology. In order to produce models that could eventually be utilized to predict space weather for operational purposes, there is also a need to run the models at speeds faster than real time. This requires high performance computing efficiency.

This paper describes the Michigan adaptive-scale MHD model and its application to the Sun-Earth system and our efforts to produce a predictive space weather code. In particular, the code we describe is built to model the entire region from the solar surface to the Earth's upper atmosphere. This requires that we address physical processes which occur over nine orders of magnitude in scale size. The code itself is described in Section II; the application of the code to simulate a coronal mass ejection is described in Section III. A companion paper in this issue, [11], provides additional details regarding the MHD code and our numerical methods as well as our recent results from simulations of the the solar wind interaction with the Earth's magnetosphere.

\section{A. Description of the Sun-Earth System}

The Sun's atmosphere, the solar corona, is so hot $\left(>10^{6} \mathrm{~K}\right)$ that in regions where the solar magnetic field is insufficiently strong, the corona undergoes a rapid expansion, filling all of interplanetary space with a superfast magnetized plasma flowing radially outward from the Sun. As this flowing plasma, which is called the solar wind, passes the Earth, it interacts strongly with the geomagnetic field, forming a bow shock upstream and 
severely compressing the field on the dayside of the Earth, and drawing it out into a long, comet-like tail on the nightside. The confined region of geomagnetic field is called the Earth's magnetosphere.

The solar wind not only confines the terrestrial magnetic field within the magnetospheric cavity, but it also transfers significant mass, momentum, and energy to the magnetosphere, as well as to the ionosphere and upper atmosphere. One dramatic consequence of this interaction between the solar wind and the magnetosphere is the generation of the aurora at high latitudes in both the northern and southern hemispheres. Another consequence is the production of a variety of complex electric current systems, ranging from a sheet of current flowing on the magnetopause boundary between the solar wind and magnetosphere, to an enormous ring of current flowing around the Earth in the inner magnetosphere, to currents flowing throughout the ionosphere and connecting along magnetic field lines to magnetospheric currents systems. Yet another result of the solarwind/magnetosphere interaction is the production of populations of very energetic particles that are stored in the magnetosphere and precipitated into the upper atmosphere. Both the electric currents and the energetic particles can have severe consequences for a number of human activities being carried out in various locations, all the way from the ground to space. It is the variation over time of these electric current systems and energetic particle populations in the geospace environment that modulates the impact of what we refer to as space weather on various human activities.

The sources of the space weather disturbances observed in the space around the Earth lies ultimately in the sun and its variability. Some geomagnetic storms can be associated with the regular 27 day appearance of long lived coronal holes which are prevalent during the declining phase of the solar cycle and at solar minimum. High speed solar wind from these holes interact with the slower solar wind to produce shock and interaction regions to produce geoeffective configurations of the interplanetary magnetic field. Other storms are the result of large coronal mass ejections in which large regions of dense material are ejected from the sun to form huge magnetic flux ropes which may interact with the Earth's magnetosphere. A major problem is to determine the evolution of solar produced disturbances as they move from the solar surface and to determine if and when they will impact the terrestrial magnetosphere, and to predict the form of the disturbance at the Earth to determine the degree of mass, momentum and energy transfer to the magnetosphere and the associated space weather which would result.

Time scales important to space weather range from minutes to decades. The longest time scales that are usually considered important to space weather are the 11-year solar activity cycle and the 22-year solar magnetic cycle (Fig. 1). Near the minimum of the solar activity cycle, the solar wind is nearly completely dominated by a quasisteady outflow, although there are occasional ejections of plasma and magnetic field from the near-equatorial sun where the plasma is generally confined by the magnetic field. These so-called coronal mass ejections (CMEs) expand into interplanetary space and become integrated into the overall solar wind flow, but they are characterized by closed magnetic field structures oriented nearly perpendicular to the ecliptic plane, in contrast to the background solar wind magnetic field, which on the average lies very nearly in the ecliptic plane. The magnetic field structures associated with these CMEs, called magnetic clouds, as well as their generally enhanced dynamic pressure $\left(\rho V^{2}\right.$, where $\rho$ is the mass density and $V$ is the bulk velocity), lead to a significantly stronger interaction with the magnetosphere than that exhibited by quiet solar wind. The major mechanism for energy coupling between the solar wind and the magnetosphere is magnetic merging on the dayside. This is substantially enhanced when the interplanetry magnetic field (IMF) contains a component antiparallel to the Earth's magnetic field, i.e., southward. The CME interaction with the magnetosphere is illustrated in Fig. 2. The magnetic cloud generated by the CME approaches the quiet magnetosphere in the top frame. In the bottom frame the cloud initiates a stronger interaction, which generates stronger magnetospheric current systems and larger, more energetic magnetospheric particle populations a phenomenon which is called a geomagnetic storm. As solar activity increases, the frequency of CMEs is substantially increased, and the "severity of space weather" is concomitantly increased.

\section{AdVANCED NumericAl MHD MODELING}

Developed with support from the NASA High Performance Computing and Communications and from NSF, the University of Michigan adaptive-scale MHD code was designed from the ground up to capitalize on modern numerical methods, solution-adaptive techniques, and massively parallel implementation. The Michigan code, whimsically called BATS-R-US for (Block Adaptive-Tree Solar-wind Roe-type Upwind Scheme), has been used successfully to simulate the environment from the solar surface to the top of the Earth's upper atmosphere.

The BATS-R-US code is based on a high-resolution scheme for the ideal MHD equations [1]. The two pieces of the high-resolution scheme are a MUSCL-type limited linear reconstruction [2]-[6] and a flux function based on an approximate solution to the MHD Riemann problem [7], [8]. This combination yields a scheme that is second-order accurate away from discontinuities, nonoscillatory near discontinuities, and robust across a wide range of plasma parameters. Details of the implementation are provided in a companion paper in this issue [11].

Solution adaptation is a powerful tool for resolving a problem with disparate length scales. By using a solution-adaptive grid, one can avoid under-resolving high-gradient regions, or, conversely, over-resolving low-gradient regions at the expense of more critical regions, and thereby save several orders of magnitude in computing resources for many problems. Length scales in the Sun-Earth system range from tens of kilometers in the ionosphere to the Earth-Sun distance $\left(1.5 \times 10^{11} \mathrm{~m}\right)$; time scales range from a few seconds near the Sun to the expansion time of the solar wind from the Sun to the Earth $\left(\sim 10^{5} \mathrm{~s}\right)$. These problems need solution-adaptive schemes-a simple Cartesian mesh would grossly under-resolve much of the problem, while over-resolving relatively uninteresting regions.

The approach to adaptation taken in the BATS-R-US code is one of self-similar adaptive blocks [9]. This approach was designed with performance on massively parallel machines in 


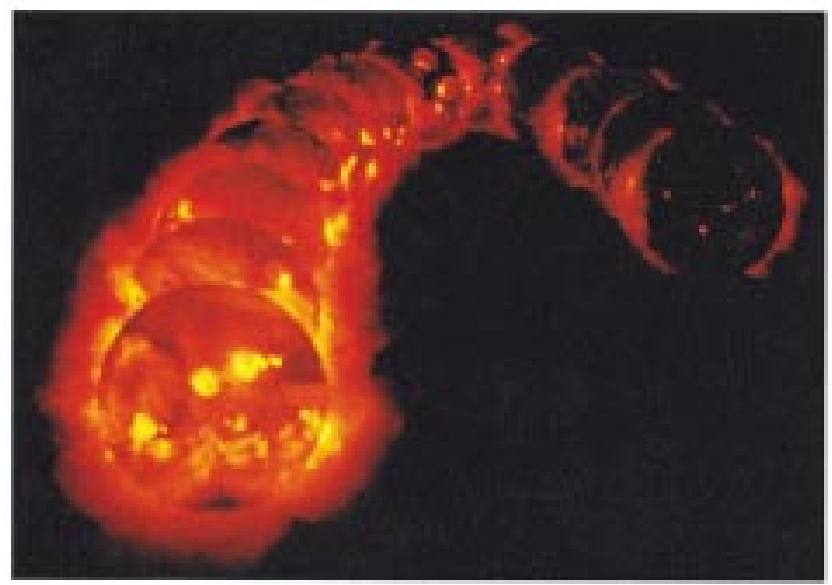

Fig. 1. The $12 \mathrm{X}$-ray images of the Sun obtained by the Yohkoh satellite between 1991 (left) and 1995 (right) at 90-day increments provide a dramatic view of how the solar corona changes from solar maximum to solar minimum. As we approach solar maximum anticipated in 2001 or 2002, the reverse progression will occur. (Figure courtesy of Lockheed Martin.)

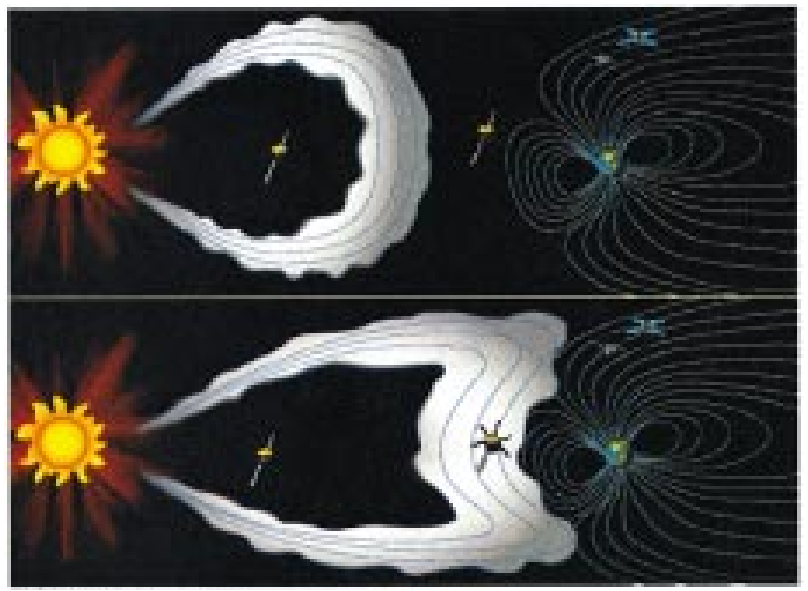

Fig. 2. The interaction of the magnetosphere with an expanding magnetic cloud. (Illustration courtesy of the NASA International Solar Terrestrial Program.)

mind. The basic unit of data is a three-dimensional block of grid cells. A block has a size that is set at run-time; a typical block might have $8 \times 8 \times 8$ cells. The data in the block are stored in $i, j, k$-indexed arrays, a data structure that is conducive to high cache performance. The code is designed to optimize serial performance on single processors of the target machine while the overall adaptive block scheme also reduces communication to provide high parallel performance. An initial grid is composed of a number of blocks, all at the same level of refinement. Then, in regions that appear under-resolved (as defined by suitable adaptation criteria), a block is refined - each of its eight octants becomes a block with the same number of cells as the original block. In regions that appear over-resolved (again, as defined by suitable adaptation criteria), eight blocks can be coalesced into one. As an example we show in Fig. 3 a two-dimensional (2-D) cut through the three-dimensional (3-D) grid taken from a calculation of a coronal mass ejection [10].

Grids like those shown in Fig. 3 go a long way toward resolving the disparate scales in a problem. Each level of refinement in the grid introduces cells that are smaller by a factor two

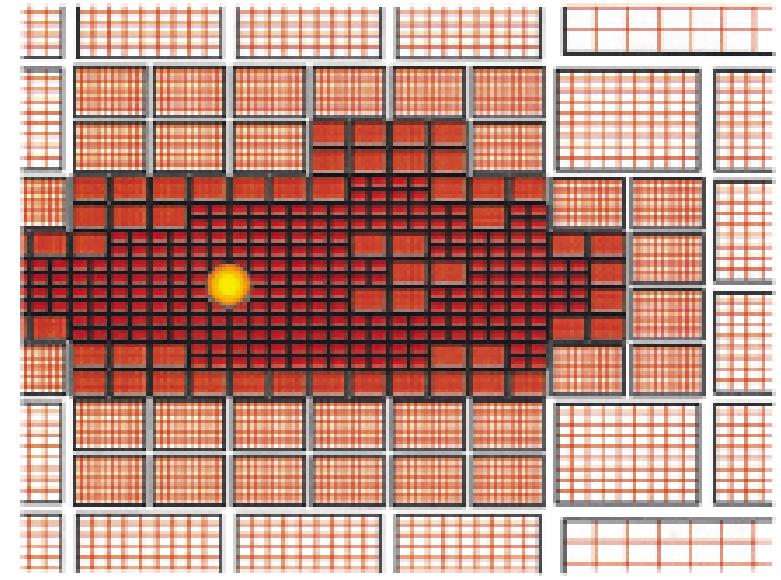

Fig. 3. Adapted grid in the simulation of a coronal mass ejection. The figure shows a $2-\mathrm{D}$ cross section of the $3-\mathrm{D}$ grid structure (cutting through the CME).

in each dimension from those one level higher in the grid. Typical calculations have 5-10 levels of refinement; some calculations have more than 20 levels of refinement. In the case of 20 levels of refinement, the finest cells on the mesh are more than one million times smaller in each dimension than the coarsest cells on a mesh.

Massively parallel machines entice users with a factor of 512 , or 1024, or even more in CPU and memory resources than single-processor machines. Capitalizing on the promise of these resources is, however, not always straightforward. In general, researchers have had very poor luck with "automatically parallelizing" their codes, or, more generally, with porting legacy codes to this class of machines. The method of domain decomposition, i.e., the partitioning of the problem by dividing the computational domain into sections and farming the separate sections off onto separate processors, is the most practical approach for many physical systems requiring the solution to partial differential equations. However, codes that were designed with single-processor computing in mind may have inherent limits on their scalability via this approach. For example, such codes may achieve speed-ups for 16 , or 32 , or 64 processors, but with additional processors, the codes not only fail to speed up, but actually slow down. These inherent limitations can arise from a variety of sources:

- underlying basic algorithms that are global in nature, resulting in high communication costs;

- underlying data structures that are expensive to partition or to update in a parallel fashion;

- underlying processes that are inherently serial.

In order to avoid any of these limitations, the BATS-R-US code was designed from the ground up with parallelism in mind. The underlying basic algorithm that was chosen is highly local in nature, resulting in low communication overhead. The adaptive-block data structures on which the code was built allow easy, natural partitioning of the data, and greatly facilitate load-balancing, a crucial element of truly scalable computing. Thus, most computation is done within a data block and all data blocks look the same to a processor, whether they are a large block or a small block. Balance and efficiency are achieved by partitioning the blocks among the available processors. The 


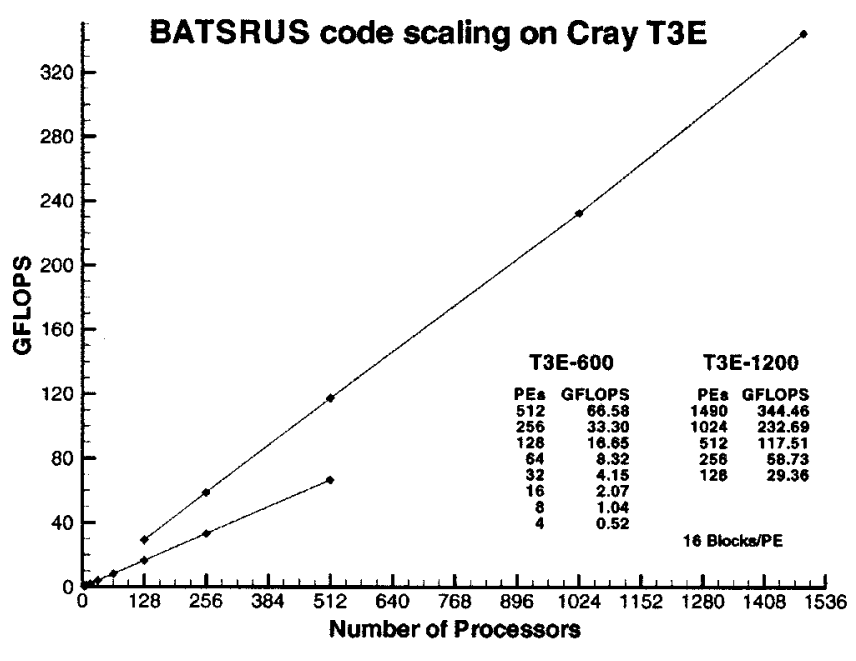

Fig. 4. Parallel scaling of the BATS-R-US code.

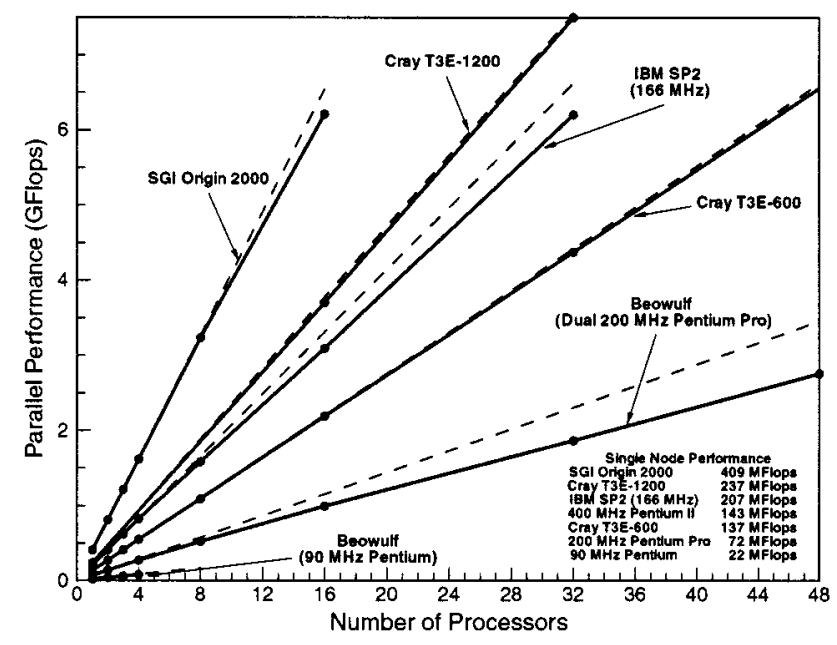

Fig. 5. Parallel performance of BATS-R-US code for a variety of parallel architectures. The dashed line indicates ideal scale-up performance based on single node performance and solid lines indicate actual performance achieved on each of the machines.

design was carried out in such a way that even the adaptation of the grid could be carried out in parallel.

The results of this bottom-up design for scalability are shown in Fig. 4. The figure shows code scaling on two different Cray T3E systems. A fixed problem size per processor of 16 blocks was used. The scale-up for both machines is nearly $100 \%$ efficient. Nearly identical results have been obtained on an IBM SP2. The code is written in Fortran 90 with message-passing via MPI, and hence is portable to a wide range of machines, from integrated shared-memory systems to networks of workstations. For each target architecture, simple single-processor measurements are used tune the size of the adaptive blocks. In Fig. 5 we show the performance obtained on a number of parallel architectures without any particular tuning.

\section{Prototype Simulation of a Coronal Mass InJeCtion FROM THE SUN TO THE EARTH}

The BATS-R-US high performance adaptive-scale MHD code has been successfully applied to the description of several solar system plasmas, including the expansion of the solar wind from the base of the solar corona to heliocentric distances well beyond the orbit of Earth and the global terrestrial magnetosphere. A schematic representation of the physical domain described by the Michigan MHD model is shown in Fig. 6.

In the solar wind simulation the source region of the solar wind (the base of the solar corona) is located at $1 R_{s}$ (solar radius). The boundary conditions describe a hot, rotating plasma reservoir with an embedded multipole field (up to octapole). The sum of the ion and electron temperatures is 3.1 million $\mathrm{K}$, the magnetic field at the solar pole and equator are $8.4 \mathrm{G}$ and 2 $\mathrm{G}$, respectively, and the plasma density is $10^{8} \mathrm{~cm}^{-3}$. Since the physical mechanism of coronal heating is still not understood, the expanding solar corona is heated by an empirical heat source function near the Sun. The specific heat ratio in the simulation is $5 / 3$, thus effects of adiabatic cooling are properly accounted for in the simulation. The resulting solar wind corresponds to solar minimum conditions. The simulation result is quite realistic: it reproduces fast solar wind $(\sim 800 \mathrm{~km} / \mathrm{s})$ above $\sim 30 \mathrm{deg}$ heliolatitude, slow $(\sim 400 \mathrm{~km} / \mathrm{s})$ solar wind near the solar equator, and realistic magnetic fields, temperatures and densities near the orbit of the Earth.

The BATS-R-US code was also used to simulate the formation and evolution of coronal mass ejections (CME) in the heliosphere. In this simulation a spatially and temporarily localized isothermal density pulse was introduced at the Sun (just above the solar equator). In this enhancement, the density and pressure are locally increased by a factor of 135 in a small region above the solar equator for a duration of 12 hours. The resulting CME rapidly moved through the near solar region and formed a structure which evolved and propagated outward passing the orbit of the Earth. Fig. 7 shows a representation of the magnetic field configuration $9 \mathrm{~h}$ after the initiation of the CME. The color code represents $\log (B)$, white lines are open magnetic field lines, magenta lines are closed with both ends connected to the sun. The density enhancement first leads to the "filling" of the closed magnetic field lines with additional plasma and subsequent expansion of the closed field region. One can see that the closed field lines become become stretched by the outward motion of the plasma. This is because the plasma $\beta$ (the ratio of the kinetic and magnetic pressures) is quite large and the magnetic field is "carried" by the outward moving plasma. Note also the decrease of magnetic field strength behind the leading edge of the outward moving disturbance.

The magnetosphere is an intricate system with the interplanetary magnetic field (IMF) playing the fundamental role in the control of the large scale topology. The magnetospheric topology in turn controls the entry of mass, momentum, energy, and magnetic flux into the magnetosphere from the solar wind. The entry of these physical quantities from the solar wind into the magnetosphere produces various transition layers, the extended geomagnetic tail and plasma sheet, various electrical current systems, charged particle precipitation, and auroral phenomena.

Global three-dimensional MHD simulations have been used to investigate the response of the magnetosphere-ionosphere system to the solar wind and to study a wide variety of processes. A few recent studies include [12]-[19]. Here, however, 


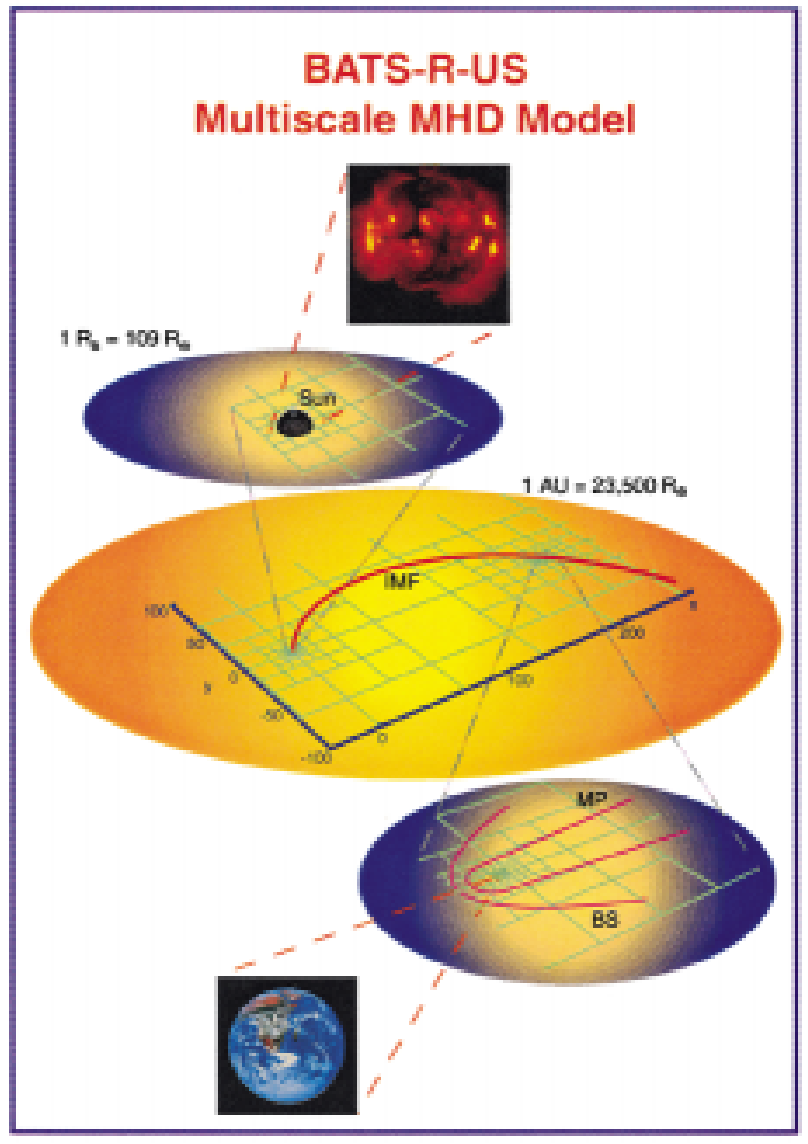

Fig. 6. The Michigan MHD Model. Note that the model spans multiple scales, from a few hundred kilometers in the ionosphere to the radius of the Earth $\left(R_{e}\right)$, to the radius of the Sun $\left(R_{s}\right)$, to the Sun-Earth distance (AU).

for the first time we have examined the dynamic response of the global magnetospheric configuration to changing solar wind conditions self-consistently simulated all the way from the Sun to $1 \mathrm{AU}$.

The solar wind changes at the Earth's location result from the rotation of the tilted multipole solar magnetic field and from the passage of the coronal mass ejection. Fig. 8 shows the time evolution of the simulated solar wind parameters as they would be measured by a spacecraft located just upstream of the subsolar bow shock. The coordinate system used here is Earth centered with the $x$ axis pointing toward the Sun, the $z$ axis pointing North and $y$ completes a right-handed system. For this simulation the Earth is not rotating and the magnetic dipole is Earthcentered and perpendicular to the Earth-Sun direction.

The first signatures of the CME are seen in the magnetic field components at around $55 \mathrm{~h}$ after initiation. The driving mass (piston) arrives around $72 \mathrm{~h}$ after initiation. The $B_{x}$ and $B_{y}$ components exhibit a significant rotation around the $y$ axis - the signature of the passage of a CME-related flux rope or magnetic cloud.

Fig. 9 shows the change of the global magnetospheric configuration during the passage of the CME. Four 3-D snapshots at $t=70.5 \mathrm{~h}, 78.5 \mathrm{~h}, 86.5 \mathrm{~h}$, and $94.5 \mathrm{~h}$. The color code represents the electric current density in the equatorial plane, solid lines show last closed magnetic field lines. The global magnetospheric configuration is primarily controlled by the IMF

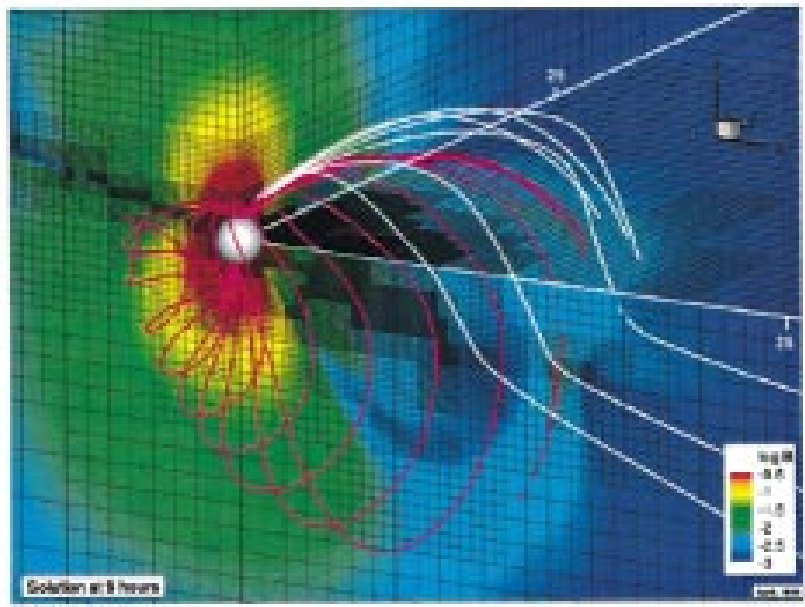

Fig. 7. Simulation of a coronal mass ejection (CME) illustrating the 3-D adapted grid structure to resolve multiple-scale phenomena. Magenta lines represent compressed closed field lines ahead of the CME, while white lines show the distorted structure of open magnetic field lines. The color code represents $\log (B)$.

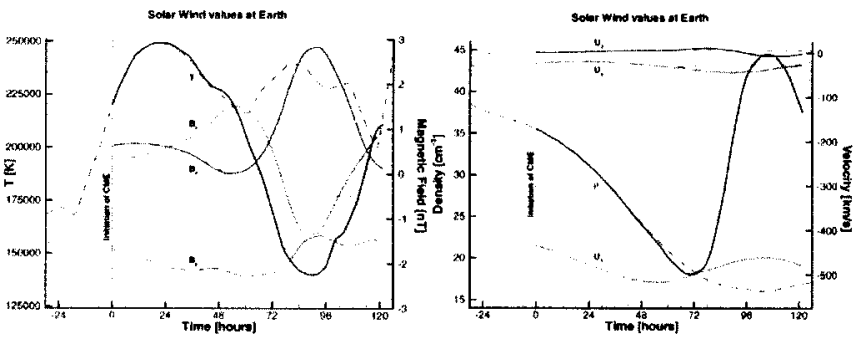

Fig. 8. Simulated solar wind parameters at the Earth-Dashed lines: without CME, solid lines: with CME.

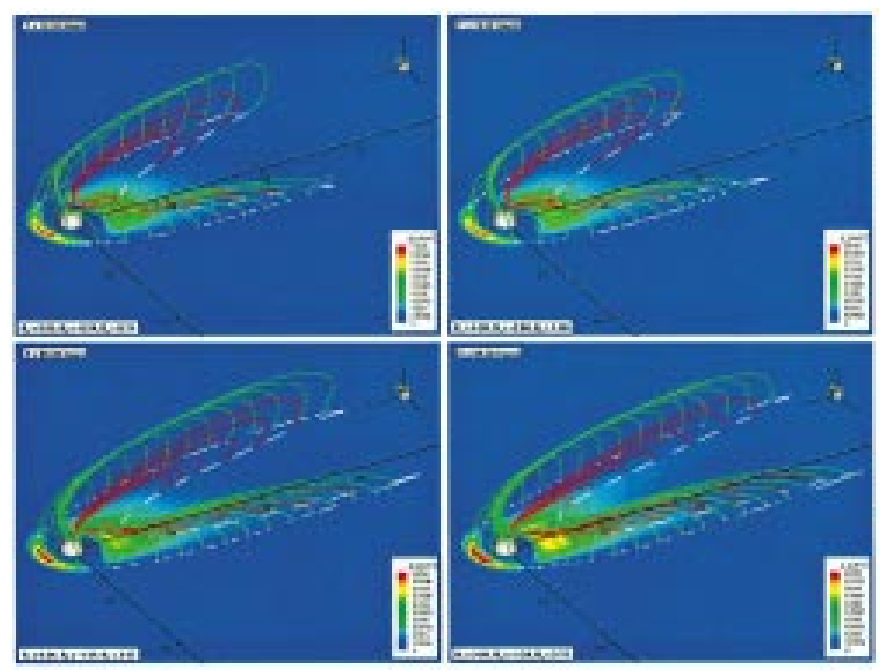

Fig. 9. The response of the magnetosphere to the CME.

$B_{z}$ component. For $B_{z}<0$ the magnetosphere exhibits an open configuration with significant dayside reconnection between IMF and geomagnetic field lines. For strong northward IMF conditions $\left(B_{z}>5 \mathrm{nT}\right)$ the magnetosphere becomes practically closed with magnetic reconnection limited to small regions above the cusps. For this CME simulation, the resulting IMF at the Earth is not particularly "geoeffective" since the IMF $B_{z}$ component is northward with intermediate values between 
0 and 3 nT. The global magnetospheric configuration does not become pure "South" or "North". However, the magnetosphere changes significantly during the event. As the solar wind dynamic pressure increases, the dayside magnetopause moves inward and the current density increases. In addition, the magnetosphere becomes narrower and the length of the magnetic wings increases from about $60 R_{E}$ to $90 R_{E}$. Overall, the energy and magnetic flux stored in the magnetosphere also increase substantially. Another interesting feature is the clockwise "twist" of the magnetosphere perpendicular to the solar wind direction. The twist, which is particularly visible in the wings, is due to the presence of the nonzero IMF $B_{y}$ component. A more detailed and complete description of the CME simulation is presented by [20].

\section{CONCLUSIONS}

Continued research into the development of high-performance numerical algorithms combined with the continued development in high-performance hardware will make it possible to achieve a predictive space weather model in the near future. The present Michigan code has been run on a Cray T3E with 512 processors and has achieved performance at about 2 times faster than real time using 2 million cells in the CME simulation for the entire Sun-Earth system. However, for operational and realistic space weather purposes, considerable improvement must be achieved. Much greater resolution, requiring approximately 10 million cells, is required, and the code must achieve 2 times faster than real time on a more modest machine, say 16 or 32 processors. Further, the code requires a number of improvements in the physics. These include a more realistic ionosphere and upper-atmospheric model, rotating Earth with a tilted dipole, as well as the inclusion of a model of the inner magnetosphere where the radiation belts and ring current are significant space weather features during magnetic storms. Thus, the challenge over the next few years is to improve the computational performance by about a factor of 100 while also including more complete and increasingly complex physical processes. Some of the anticipated performance improvement will be accomplished by improvements in computational hardware capabilities, and further improvement will be accomplished by improvements in our numerical algorithm technology and software design.

\section{REFERENCES}

[1] K. G. Powell, P. L. Roe, T. J. Linde, T. I. Gombosi, and D. L. DeZeeuw, "A solution-adaptive upwind scheme for ideal magnetohydrodynamics," J. Comput. Phys., 1999.

[2] B. van Leer, "Toward the ultimate conservative difference scheme. I. The quest of monoticity," Lecture Notes Phys., vol. 18, 1973.

[3] - "Toward the ultimate conservative difference scheme. II. Monoticity and conservation combined in a second-order scheme," $J$. Comput. Phys., vol. 14, 1974.

[4] - "Toward the ultimate conservative difference scheme. III. Upstream-centered finite-difference schemes for ideal compressible flow," J. Comput. Phys., vol. 23, 1977.

[5] - "Toward the ultimate conservative difference scheme. IV. A new approach to numerical convection," J. Comput. Phys., vol. 23, 1977.
[6] - "Toward the ultimate conservative difference scheme. V. A second-order sequel to Godunov's method," J. Comput. Phys., vol. 32, pp. 101-136, 1979.

[7] K. G. Powell, "An approximate Riemann solver for magnetohydrodynamics (that works in more than one dimension)," Tech. Rep. 94-24, ICASE, Langley, VA, 1994.

[8] T. J. Linde, "A three-dimensional adaptive multifluid MHD model of the heliosphere," Ph.D. dissertation, Univ. Michigan, Ann Arbor, MI, May 1998.

[9] Q. F. Stout, D. L. DeZeeuw, T. I. Gombosi, C. P. T. Groth, H. G. Marshall, and K. G. Powell, "Adaptive blocks: A high-performance data structure," in Proc. Supercomputing'97, 1997.

[10] _ "Adaptive parallel computation of a grand-challenge problem: Prediction of the path of a solar coronal mass ejection," in Proc. Supercomputing'98, 1998.

[11] D. L. DeZeeuw, T. I. Gombosi, C. P. T. Groth, K. G. Powell, and Q. F. Stout, "An adaptive mhd method for global space weather simulations," IEEE Trans. Plasma Sci., vol. 28, Dec. 2000.

[12] P. Janhunen, "GUMICS-3: A global ionosphere-magnetosphere coupling simulation with high ionospheric resolution," in Proc. ESA 1996 Sympo. Environment Modeling Space-Based Appl., ESA SP-392, 1996.

[13] P. Janhunen and H. E. J. Koskinen, "The closure of Region-1 fieldaligned current in MHD simulation," Geophys. Res. Lett., vol. 24, no. 11, pp. 1419-1422, June 1997.

[14] J. Raeder, J. Berchem, M. Ashour-Abdalla, L. A. Frank, W. R. Paterson, K. L. Ackerson, S. Kokubun, T. Yamamoto, and J. A. Slavin, "Boundary layer formation in the magnetotail: Geotail observations and comparisons with a global MHD simulation," Geophys. Res. Lett., vol. 24, pp. 951-954, 1997.

[15] R. M. Winglee, "Regional particle simulations and global two-fluid modeling of the magnetospheric current system," in Cross-Scale Coupling in Space Plasmas, J. L. Horwitz, N. Singh, and J. L. Burch, Eds: AGU, 1995, vol. 93, p. 71. Geophysical Mongraph.

[16] R. M. Winglee, V. O. Papitashvili, and D. R. Weimer, "Comparison of the high-latitude ionospheric electrodynamics inferred from global simulations and semiempirical models for the January 1992 GEM campaign," J. Geophys. Res., vol. 102, pp. 26 961-26977, 1997.

[17] T. I. Gombosi, D. L. DeZeeuw, C. P. T. Groth, K. G. Powell, and P. Song, "The length of the magnetotail for northward IMF: Results of 3D MHD simulations," in Phys. Space Plasmas (1998), T. Chang and J. R. Jasperse, Eds: MIT Press, 1998, vol. 15, pp. 121-128.

[18] T. I. Pulkkinen, D. N. Baker, M. Wiltberger, C. Goodrich, R. E. Lopez, and J. G. Lyon, "Pseudobreakup and substorm onset: Observations and MHD simulations compared," J. Geophys. Res., vol. 103, no. A7, pp. 14 847-14 854, July 1998.

[19] W. W. White, G. L. Siscoe, G. M. Erickson, Z. Kaymaz, N. C. Maynard, K. D. Siebert, B. U. Ö. Sonnerup, and D. R. Weimer, "The magnetospheric sash and the cross-tail S," Geophys. Res. Lett., vol. 25, no. 10, pp. 1605-1608, May 1998.

[20] C. P. T. Groth, D. L. DeZeeuw, T. I. Gombosi, and K. G. Powell, "Global $3 \mathrm{~d}$ mhd simulation of a space weather event: Cme formation, interplanetary propagation, and interaction with the magnetosphere," J. Geophys. Res., 1999.
C. Robert Clauer received the Ph.D. degree in geophysics and space physics from the University of California, Los Angeles in 1980.

He was a Research Associate and Senior Research Associate at Stanford University, undertaking some of the first experiments utilizing the Sondrestrom Incoherent Scatter Radar in Søndre Strømfjord, Greenland. Prior to joining the faculty at the University of Michigan in 1991, he was a Senior Research Physicist at SRI International. From March 1996 to March 1998, he was the Program Director for Magnetospheric Physics at the National Science Foundation while on leave from the University of Michigan. He is the author or co-author of over 80 scientific publications and has given over 160 scientific presentations.

Dr. Clauer has participated on many national and international scientific advisory committees, been the convenor of several international scientific meetings, is an Associate Editor of the Journal of Geophysics—Space Physics, and received the University of Michigan College of Engineering Outstanding Research Achievement Award in 1992 and has received the National Science Foundation Director's Award for Collaborative Integration in 1998. 
Tamas I. Gombosi was educated in theoretical physics at the Lóránd Eötvös University in Budapest, Hungary. He did his post-doctoral research at the Space Research Institute in Moscow under the direction of Dr. Konstantin Gringauz. $\mathrm{He}$ is author or co-author of over 150 refereed publications and gave over 200 scientific presentations. He published two graduate level textbooks: Gaskinetic Theory (Cambridge, 1994) and Physics of the Space Environment (Cambridge, 1998)

In 1987 he joined the University of Michigan where he is presently Professor of Space Science and Professor of Aerospace Engineering. His research interests span from the physics of comets, through heliospheric plasmas to planetray plasma environments.

Dr. Gombosi served as Editor-in-Chief of the Journal of Geophysical Research-Space Physics (1992-1997). He also served on a large number of national and international scientific committees, including the Committee on Solar and Space Physics of the Space Studies Board (National Academy of Sciences), and is presently Chairman of Commission D (Space Plasmas) of COSPAR. He is the recipient of several scientific awards. He is an elected Member of the International Academy of Astronautics and a Fellow of the American Geophysical Union.

Darren L. De Zeeuw received the Ph.D. degree in Aerospace engineering and scientific computing from the University of Michigan in 1993.

Upon completion of his dissertation work, he joined the Space Physics Research Laboratory to work with the space plasma simulation group to develop a magnetohydrodynamic (MHD) model based on his dissertation work.

Aaron J. Ridley received the B.S. degree in physics from Eastern Michigan University in 1992 and Ph.D. in atmospheric and space sciences from the University of Michigan in 1997. While in graduate school, he received a fellowship from the National Center for Atmospheric Research's High Altitude Observatory, where he studied nonsteady-state global ionospheric convection.

From 1997 to 1999, he worked as a Postdoctoral Researcher and a Research Scientist at Southwest Research Institute. His studies there focused on the coupling of the magnetosphere-ionosphere system, and real-time data assimilation techniques. Recently, he has moved back to the University of Michigan, where he is working on modeling the coupling of the magnetosphere and ionosphere. He has published approximately 10 papers in the field of space physics. He is a member of the American Geophysical Union.

Kenneth G. Powell received the doctorate degree in aeronautics and astronautics from MIT in 1987, where Dr. Earl Murman was his thesis advisor.

After leaving MIT, he joined the faulty in the Aerospace Department at the University of Michigan. He has branched out into the research areas of solutionadaptive schemes, genuinely multidimensional schemes for compressible flows, and, most recently, numerical methods for mag-netohydrodynamics.

He was a National Science Foundation Presidential Young Investigator from 1988-1994. He was a recipient of a NASA Group Achievement Award in 1989 for his work in vortical flows.

Bram van Leer has academic degrees in astronomy and astrophysics, which he studied in Leiden with foremost Dutch astronomers J.H. Oort and H.C. van de Hulst.

After a short interlude teaching numerical mathematics in Delft (1982-86) he became a full Professor in the Department of Aerospace Engineering at the University of Michigan. His research interest currently centers on convergence acceleration of steady-flow computations, in particular, the development of truly effective multigrid methods for high-Reynolds-number flows, using local preconditioning to remove the stiffness of the governing equations.
Quentin F. Stout received the Ph.D. degree in mathematics, but for over 15 years he has worked in the areas of parallel computing and parallel algorithms. His professional activities in parallel computing include co-authoring the book Parallel Algorithms for Regular Architectures: Meshes and Pyramids, 1996 (MIT Press); writing over 100 articles and book chapters; editing three parallel computing journals and a book on reconfigurable architectures; co-chairing the program for three international conferences (and being on the program committee of many others); lecturing at NATO Advanced Study Institutes; and giving tutorials on parallel computing at numerous conferences, corporations, and government agencies. He is the Director of the University of Michigan's Center for Parallel Computing, which is part of the National Science Foundation's newly created National Partnership for Advanced Computing Infrastructure (NPACI).

Clinton P. T. Groth completed his undergraduate work at the University of British Columbia in 1983. He subsequently obtained both a Master's degree and his doctorate in aerospace engineering from the University of Toronto in 1987 amd 1993, respectively.

Following a NSERC Postdoctoral Research Fellowship at the University of Michigan, Department of Aerospace Engineering, he joined the Space Physics Research Laboratory at the University of Michigan in 1996 as an Assistant Research Scientist. He has only recently joined the faculty at the University of Toronto Institute for Aerospace Studies as an Assistant Professor. His research interests lie in the area of computational fluid dynamics (CFD) and he has specialized in the application of upwind finite-volume schemes for hyperbolic conservation laws, adaptive mesh refinement techniques, parallel solution methods, the development of flux functions based on the solution of the Riemann problem, the computation of nonequilibrium, rarefied, and magnetized flows with strong shocks, and the development of generalized transport models and solution methods following from kinetic theory.

$\mathrm{He}$ is a member of the American Institute of Aeronautics and Astronautics, American Geophysical Union, Society for Industrial and Applied Mathematics, and Canadian Aeronautics and Space Institute. In recognition of his graduate achievements, Prof. Groth was the 1991 recipient of the G. N. Patterson Award and a 1992-93 University of Toronto Governor General's Gold Medal.

Thomas E. Holzer received the B.A. degree in physics from Pomona College in 1965 and the Ph.D. degree in applied physics from the University of California, San Diego in 1970

After a NATO Postdoctoral Fellowship at Imperial College and an NRC Resident Research Associateship at NOAA's Aeronomy Laboratory, he joined the High Altitude Observatory (HAO) of the National Center for Atmospheric Research in 1973. He became a Senior Scientist in 1978, served as Director of HAO from 1990 to 1995, and is now again engaged in full-time research. He has also been affiliated with the Department of Astrophysical and Planetary Sciences at the University of Colorado since 1972 and has been an Adjoint Professor since 1981. He has published more than 100 papers in a wide range of areas of space physics and astrophysics. His current research interests are focused on the heating of the solar corona, the acceleration of the solar wind, the origin of solar coronal mass ejections, and plasma flow in the terrestrial ionosphere-magnetosphere system.

Dr. Holzer received the James B. Macelwane Award of the American Geophysical Union in 1978, is a Fellow of the American Geophysical Union, and was inducted into the Norwegian Academy of Science and Letters in 1995. 